\title{
POST-NEWTONIAN APPROXIMATIONS AND EQUATIONS OF MOTION OF GENERAL RELATIVITY
}

\author{
GERHARD SCHÄFER \\ Max-Planck-Arbeitsgruppe Gravitationstheorie \\ Friedrich-Schiller-Universität, 07743 Jena, Germany \\ E-mail: gos@gravi.physik.uni-jena.de
}

\begin{abstract}
A post-Newtonian approximation scheme for general relativity is defined using the Arnowitt-Deser-Misner formalism. The scheme is applied to perfect fluids and point-mass systems. The two-body point-mass Hamiltonian is given explicitly up to the post ${ }^{2.5}$-Newtonian order.

1. Introduction. The Newtonian theory of gravity describes the motion of celestial bodies with remarkably good precision. Only very precise measurements show deviations from the Newtonian description. The Newtonian dynamics is therefore strongly expected to define an excellent starting point for an approximation scheme of general relativity, in situations for which the velocities of the bodies are small compared to the velocity of light $\left((v / c)^{2}<<1\right)$. On the other, theoretical side, $(3+1)$-formalisms of general relativity with elliptic constraint and hyperbolic evolution equations are closest to the elliptic-type Newtonian theory. Therefore it seems natural to define post-Newtonian approximation schemes in those formalisms. In the following we shall choose the Arnowitt-Deser-Misner (ADM) Hamiltonian formalism of general relativity, [3], as one of the best-known and fully developed $(3+1)$-formalisms and define a post-Newtonian approximation scheme in that context. For post-Newtonian approximation schemes in other settings, e.g. see [8], [9]; for a critical investigation of the Newtonian limit in the theory of general relativity, see $[15]$.
\end{abstract}

2. The ADM Hamiltonian of general relativity. In the ADM canonical formalism of general relativity the Hamiltonian of the total system, $H$, is the most important quantity. The Hamiltonian is defined on space-like three-dimensional, asymptotically flat Cauchy hypersurfaces which in their asymptotic regions are covered by cartesian coordinate systems. The Hamiltonian is conserved in time and it contains the full information

1991 Mathematics Subject Classification: 83C10, 83C25.

The paper is in final form and no version of it will be published elsewhere. 
about the field-plus-matter dynamics. $H$ is given by, [3], [24],

$$
H=\int d^{3} x\left(N h+N^{i} h_{i}\right)+\frac{c^{4}}{16 \pi G} \oint_{\infty} d^{2} s_{i}\left(\gamma_{i j, j}-\gamma_{j j, i}\right),
$$

where

$$
h=\frac{(16 \pi G)^{2}}{c^{6}} \pi^{i j} G_{i j k l} \pi^{k l}-\gamma^{1 / 2(3)} R+\frac{16 \pi G}{c^{4}} \gamma^{1 / 2} n_{\mu} T^{\mu \nu} n_{\nu}
$$

denotes the so-called super Hamiltonian and

$$
h_{i}=-2 \gamma_{i k} D_{j} \pi^{k j}-\pi_{i}
$$

the so-called super momentum.

The independent gravitational field variables in the Hamiltonian are the metric components of the space-like hypersurfaces, $\gamma_{i j}:=g_{i j}=g_{j i}\left(x^{i}\right.$, with $i=1,2,3$, are spatial coordinates in the surfaces and $x^{0}=c t$ is labelling the surfaces, $\mathrm{c}$ denotes the velocity of light and $t$ is a time parameter), and their canonical conjugate momenta, $\pi^{i j}$. The lapse and shift functions, $N:=\left(-g^{00}\right)^{-1 / 2}$ and $N^{i}:=\gamma^{i j} g_{0 j}$, respectively, are Lagrangian multipliers. $\gamma,{ }^{(3)} R, \gamma^{i j}$, and $D_{j}$ denote, respectively, the determinant of the 3-metric, the curvature scalar of a hypersurface, the inverse 3-metric, and the 3-dimensional covariant derivative. $G_{i j k l}:=\frac{1}{2} \gamma^{-1 / 2}\left(\gamma_{i k} \gamma_{j l}+\gamma_{i l} \gamma_{j k}-\gamma_{i j} \gamma_{k l}\right)$ can be regarded as a contravariant metric in a 6 -dimensional pseudo-Riemannian manifold with signature $+4,[14] . \quad G$ is the Newtonian gravitational constant. The future-oriented unit normal vector of a hypersurface is denoted by $n^{\mu}$. T $T^{\mu \nu}$ is the symmetric stress-energy tensor of the matter. The momentum density of the matter is given by $\pi_{i}=-c^{-1} \gamma^{1 / 2} n_{\mu} T_{i}^{\mu}=c^{-1}(-g)^{1 / 2} T_{i}^{0}$. The surface integral in equation (1) is taken over the 2 -sphere at infinity in a Cauchy hypersurface.

If we take as model for the matter a barotropic perfect fluid, the stress-energy tensor takes the simple form

$$
T^{\mu \nu}=(e+p) u^{\mu} u^{\nu}+p g^{\mu \nu},
$$

where $e$ denotes the energy density in the rest frame of the fluid and $p$ its pressure. The time-like 4 -velocity of the fluid, $u^{\mu}$, is normalized according to $u^{\mu} u^{\nu} g_{\mu \nu}=-1 . g_{\mu \nu}$ is the space-time metric $(\mu, \nu=0,1,2,3$; metric signature: +2$)$. The conservation law for the baryonic mass number has the form $D_{\mu}\left(\varrho u^{\mu}\right)=0$, where $\varrho$ denotes the baryonic mass density and $D_{\mu}$ the 4-dimensional covariant derivative. The conserved baryonic mass number, $m^{*}$, reads $m^{*}=\int d^{3} x \varrho^{*}$, where, by definition, $\varrho^{*}=-\gamma^{1 / 2} \varrho n_{\mu} u^{\mu}=(-g)^{1 / 2} \varrho u^{0}$ holds. $\varrho^{*}$ fulfils the continuity equation $\partial_{t} \varrho^{*}+\partial_{i}\left(\varrho^{*} v^{i}\right)=0$, where the velocity $v^{i}$ is defined by $v^{i}=c u^{i} / u^{0}$. Introducing the specific energy per unit baryonic mass, $\Pi$, which depends on $\varrho$ only, the energy density and the pressure are given by $e=\varrho c^{2}\left(1+\Pi / c^{2}\right), p=$ $\varrho^{2} d \Pi / d \varrho$.

If $\varrho^{*}$ and $\pi_{i}$ are chosen as dynamical variables for the fluid, the equations of motion read,

$$
\begin{gathered}
\frac{\partial \varrho^{*}}{\partial t}=-\partial_{i}\left(\frac{\delta H}{\delta \pi_{i}} \varrho^{*}\right), \\
\frac{\partial \pi_{i}}{\partial t}=-\partial_{s}\left(\frac{\delta H}{\delta \pi_{s}} \pi_{i}\right)-\partial_{i}\left(\frac{\delta H}{\delta \pi_{s}}\right) \pi_{s}-\partial_{i}\left(\frac{\delta H}{\delta \varrho^{*}}\right) \varrho^{*},
\end{gathered}
$$


where $(\delta \ldots) /(\delta \ldots)$ denotes the Fréchet derivative and $\partial_{i}:=\partial / \partial x^{i} . \quad \frac{\delta H}{\delta \pi_{i}}=v^{i}$ defines a Legendre transformation to a Lagrangian description with $\varrho^{*}$ and $v^{i}$ as independent fluid variables, $L=\int d^{3} x\left(\pi_{i} v^{i}+\pi^{i j} \partial_{t} \gamma_{i j}\right)-H$.

The equations of motion, (5) and (6), can be written also in terms of Poisson brackets, so-called 'Lie-Poisson' (-'Kirillov-Kostant') brackets which are intimately related with the 3-dimensional diffeomorphism group of the Cauchy hypersurfaces, e.g. see [1], [17].

The Einstein field equations split into constraint equations,

$$
\begin{gathered}
h=0 \text { (Hamiltonian constraint), } \\
h_{i}=0 \text { (momentum constraint), }
\end{gathered}
$$

and evolution equations,

$$
\begin{gathered}
\frac{\partial \gamma_{i j}}{\partial t}=\frac{\delta H}{\delta \pi^{i j}}, \\
\frac{\partial \pi^{i j}}{\partial t}=-\frac{\delta H}{\delta \gamma_{i j}}
\end{gathered}
$$

The Hamiltonian constraint is a generalization of the theorema egregium by Gauss and the momentum constraint generalizes the Codazzi theorem of vanishing covariant divergence of the extrinsic curvature. It is an important property of the constraint equations that they are conserved in time, i.e. they commute with the Hamiltonian (1).

Within the full theory we may choose the following four coordinate conditions, [2], corresponding to the Hamiltonian and momentum constraint, respectively, (i.e. the coordinate conditions are, respectively, not invariant against the transformations generated by the super Hamiltonian and super momentum, e.g. see [30]),

$$
\begin{gathered}
\pi^{i j} \delta_{i j}=0, \\
\gamma_{i j}=\left(1+\frac{2 \pi G}{c^{2}} \phi\right)^{4} \delta_{i j}+\frac{16 \pi G}{c^{4}} h_{i j}^{T T} .
\end{gathered}
$$

$h_{i j}^{T T}$, apart from a factor, denotes the part of the 3-metric which is transverse and traceless in the flat-space metric $\delta_{i j}$ (Kronecker's $\delta$ ). The canonical conjugate to $\left(16 \pi G / c^{4}\right) h_{i j}^{T T}$ will be denoted by $c^{-2} p^{T T i j}$, i.e. $p^{T T i j}=c^{2} \pi^{T T i j}$, where $\pi^{T T i j}$ is the transverse-traceless part of $\pi^{i j}$. The fields $h^{T T}\left(:=h_{i j}^{T T}\right)$ and $p^{T T}\left(:=p^{T T i j}\right)$ represent the "true" degrees of freedom of the gravitational field. The fields $h^{T T}$ and $p^{T T}$ are scaled in such a way that, in the limit of vanishing $1 / c$, they remain finite if generated by a slowly moving source $(v / c \rightarrow 0)$.

The application of the coordinate conditions, (11) and (12), to the field equations, (9) and (10), result in elliptic-type equations for the lapse and shift functions (notice that the coordinate conditions (12) are equivalent to $3 \partial_{j} \gamma_{i j}-\partial_{i} \gamma_{j j}=0$ ). These equations guarantee that the coordinate conditions hold at any instant of time during the dynamical evolution, i.e. they commute with the Hamiltonian (1). Therefore, like the constraint equations which determine through elliptic-type equations $\phi$ and the vector part of the flat-space-traceless $\pi^{i j}\left(\pi^{i j}=\pi^{T T i j}+\partial_{i} \tau^{j}+\partial_{j} \tau^{i}-\delta_{i j} \partial_{l} \tau^{l}+\nabla^{-2} \partial_{i} \partial_{j} \partial_{l} \tau^{l}\right)$, the equations for the lapse and shift functions can be solved for all instants of time. 
The evolution equations for $h^{T T}$ and $p^{T T}$ read,

$$
\begin{gathered}
\frac{\partial h^{T T}}{\partial t}=\frac{c^{6}}{16 \pi G} \frac{\delta H_{r e d}}{\delta p^{T T}} \\
\frac{\partial p^{T T}}{\partial t}=-\frac{c^{6}}{16 \pi G} \frac{\delta H_{r e d}}{\delta h^{T T}} .
\end{gathered}
$$

$H_{\text {red }}$ denotes $H$ if restricted to the space of functions which fulfil the constraint equations and the coordinate conditions, i.e.

$$
H_{r e d}=-c^{2} \int d^{3} x \Delta \phi=-c^{2} \oint_{\infty} d^{2} s_{i} \partial_{i} \phi=H\left[\varrho^{*}, \pi, h^{T T}, p^{T T}\right],
$$

$\left(\pi:=\pi_{i}\right)$.

Now, in the equations of motion for the perfect fluid, (5) and (6), $H$ gets substituted by $H_{\text {red }}$. Notice also the reduced Lagrangian: $L_{r e d}=\int d^{3} x\left(\pi v+16 \pi G p^{T T} \partial_{t} h^{T T} / c^{6}\right)-H_{r e d}$, $\left(v:=v^{i}\right)$.

For completeness we add the expressions for the total linear momentum and angular momentum of the matter system, $\int d^{3} x \pi_{i}$ and $\int d^{3} x \epsilon_{i j k} x^{j} \pi_{k}$, respectively, where $\epsilon_{i j k}$ denotes the Levi-Civita anti-symmetric tensor.

After solving the constraint equations, the metric components $g_{\mu \nu}$ are functionals of the independent variables $\varrho^{*}, \pi, h^{T T}$, and $p^{T T}$.

3. Post-Newtonian approximations in the ADM formalism. A series expansion in powers of $1 / c$ of the metric components and of the equations of motion of the matter is called a post-Newtonian expansion of the theory.

In terms of the independent field and matter variables we are able to define a postNewtonian expansion of the metric tensor, [28],

$$
\begin{aligned}
c^{2} g_{00}(t, x) & =-c^{2}+\sum_{n=0}^{\infty}\left(\frac{1}{c^{2}}\right)^{n} h_{00}^{[n]}\left(x ;\left[\varrho^{*}(t), \pi(t), h^{T T}(t), p^{T T}(t)\right]\right), \\
c g_{0 i}(t, x) & =\sum_{n=1}^{\infty}\left(\frac{1}{c^{2}}\right)^{n} h_{0 i}^{[n]}\left(x ;\left[\varrho^{*}(t), \pi(t), h^{T T}(t), p^{T T}(t)\right]\right), \\
g_{i j}(t, x) & =\delta_{i j}+\sum_{n=1}^{\infty}\left(\frac{1}{c^{2}}\right)^{n} h_{i j}^{[n]}\left(x ;\left[\varrho^{*}(t), \pi(t), h^{T T}(t), p^{T T}(t)\right]\right),
\end{aligned}
$$

$\left(x:=x^{i}\right)$. Here, the $h_{\mu \nu}^{[n]}$ are the metric components at the post ${ }^{n}$-Newtonian order, i.e. $n$-th post-Newtonian approximation. The components are determined by solely elliptic-type equations in flat space. $h_{00}^{[0]}$ is minus two times the usual Newtonian potential. The field variables $h^{T T}$ and $p^{T T}$ enter the metric components at the post ${ }^{2}$ - and post ${ }^{3}$-Newtonian approximations, respectively. As $h^{T T}$ and $p^{T T}$ vanish for spherically symmetric processes, the post-Newtonian power expansions will exist in those situations to all orders.

The reduced Hamiltonian takes now the form,

$$
H_{\text {red }}=c^{2} \int d^{3} x \varrho^{*}+\sum_{n=0}^{\infty}\left(\frac{1}{c^{2}}\right)^{n} H_{r e d}^{[n]}\left[\varrho^{*}, \pi, h^{T T}, p^{T T}\right] .
$$


In the following we shall always suppress the baryonic mass-energy $c^{2} \int d^{3} x \varrho^{*}$ in the reduced Hamiltonian as it drops out completely from the dynamics.

$H_{r e d}^{[0]}$ is the Hamiltonian of the Newtonian theory. For our matter model, it simply reads, [26],

$$
H_{r e d}^{[0]}=\int d^{3} x \varrho^{*} \Pi\left(\varrho^{*}\right)+\frac{1}{2} \int d^{3} x \frac{\pi^{2}}{\varrho^{*}}-\frac{1}{2} \iint d^{3} x d^{3} y \frac{\varrho^{*}(t, x) \varrho^{*}(t, y)}{|x-y|},
$$

where $|x-y|$ denotes the usual Euclidean distance between $x$ and $y$.

$H_{\text {red }}^{[0]}+\frac{1}{c^{2}} H_{\text {red }}^{[1]}$ is the post ${ }^{1}-$ Newtonian Hamiltonian. Up to this order no field variables are involved. At the post ${ }^{2}-$ Newtonian order, $h^{T T}$ is entering; at the post ${ }^{3}$-Newtonian level, $p^{T T}$ is coming in, i.e. at this level the gravitational field starts evolving in time, [27]. It can be shown that at the post ${ }^{2}$-Newtonian order the field variable $h^{T T}$ may be substituted in the Hamiltonian completely by matter variables; see the post ${ }^{2}$-Newtonian two-body Hamiltonian below.

A post-Newtonian expansion of the equations of motion, (5) and (6), is immediately achieved by use of the Hamiltonian (19).

To further discuss the time evolution of the field variable $h^{T T}$, we develop its field equation into a second (differential-)order form wave equation,

$$
\left(\nabla^{2}-\frac{\partial^{2}}{c^{2} \partial t^{2}}\right) h^{T T}(t, x)=\sum_{n=0}^{\infty}\left(\frac{1}{c^{2}}\right)^{n} D_{[n]}^{T T}\left(x ;\left[\varrho^{*}(t), \pi(t), h^{T T}(t), \partial_{t} h^{T T}(t)\right]\right) .
$$

The functional $D_{[0]}^{T T}$ depends on $\varrho^{*}$ and $\pi$ only. It contains the full information about the leading order quadrupole radiation. The whole information of the post ${ }^{1}$-Newtonian wave generation is incorporated in $D_{[0]}^{T T}+\frac{1}{c^{2}} D_{[1]}^{T T} \cdot D_{[1]}^{T T}$ depends on $\varrho^{*}$ and $\pi$, and on $h^{T T}$ linearly.

The formal solution of equation (21), assuming the condition of no incoming radiation from light-like past infinity $(t+r / c=b, t \rightarrow-\infty, b=$ constant $)$, uniquely reads,

$$
h^{T T}(t, x)=-\frac{1}{4 \pi} \sum_{n=0}^{\infty}\left(\frac{1}{c^{2}}\right)^{n} \int \frac{d^{3}}{|x-y|} D_{[n]}^{T T}\left(y ;\left[\varrho^{*}(u), \pi(u), h^{T T}(u), \partial_{u} h^{T T}(u)\right]\right),
$$

where $u=t-|x-y| / c$ is the time at the source point $y$, represented in terms of the retarded time $t$ at the field point $x$. Obviously, a uniform expansion of equation (22) in powers of $1 / c$ is not feasible in general.

The solution of $p^{T T}$ is obtained by solving the equation (13) for $p^{T T}$ (Legendre transformation for the gravitational field).

The solution (22) exists if its energy (numerical value of the Hamiltonian) is finite. For this it is sufficient to check that the numerical value of the free-field part of the Hamiltonian, $H^{r a d}$, is finite. $H^{r a d}$ is given by

$$
H_{\text {red }}^{\text {rad }}=\frac{16 \pi G}{c^{4}} \int d^{3} x\left[\frac{1}{4}\left(\nabla h^{T T}\right)^{2}+\frac{1}{c^{2}}\left(p^{T T}\right)^{2}\right] .
$$

An approximate treatment of equation (22) has shown that the gravitational field generated from freely infalling bodies has finite energy, to leading order at least, [25].

The explicit solution (22) can be constructed order by order in the ordering in $n$. At any finite order a well-defined approximate solution can be expected if the starting 
approximate solution has finite energy. Nothing is known, however, about the convergence of the series of approximate solutions if the independent variables depend on time. If they do not depend on time, a convergent series can be expected, e.g. for stationary rotating bodies. In stationary situations the solution for $h^{T T}$ is fully expressible in powers of $(1 / c)^{2}$ as the independent variables do not depend on time. In non-stationary situations one can try to expand the arguments of the independent variables in powers of $1 / c$, either in the near zone, if one is interested in the equations of motion for the matter, or in the far zone, if one is interested in the radiation. In the near zone, for fixed $t$, this expansion is an expansion in powers of $|x-y| / c$, in the far zone, for fixed $t-r / c$, it is an expansion in powers of $y \cdot n / c$ (notice: $u=t-r / c+y \cdot n / c+O\left(r^{-1}\right)$ ), where $n=x / r, r=|x|)$. However, already at the post ${ }^{4}$-Newtonian order, i.e. expansion of $D_{[0]}^{T T}$ to the order $c^{-4}$, those power-series expansions break down. If one is interested in information beyond post ${ }^{3.5}$-Newtonian order one has to treat the independent field varibles in their non-1/c-expanded form. Nevertheless, as the non-analytic terms in $1 / c$ belong more to the "phases" than to the "amplitudes" of the independent field variables, it may happen that some final expressions are fully expandable in powers of $1 / c$ if one chooses the phases correctly, i.e. if the flat-space time parameter of the source, $t-\frac{r}{c}$, is shifted to the true-space time parameter, $t-\frac{r}{c}-\frac{2 M G}{c^{3}} \ln \left(\frac{r}{c \alpha}\right)$, where $M$ denotes the total mass of the system and where the parameter $\alpha$ fixes the coordinate system in the far zone, e.g. see [7]. Furthermore, to really know that the radiation comes from the source only, the no-incoming radiation condition has to be checked with the true light-like past infinity $\left(t+\frac{r}{c}+\frac{2 M G}{c^{3}} \ln \left(\frac{r}{c \alpha}\right)=b, t \rightarrow-\infty\right)$.

4. Energy balance considerations. Let us decompose the Hamiltonian into a part which depends on matter variables only, but without the baryonic mass-energy, $H_{r e d}^{\text {mat }}$, a part which depends on field variables only, $H_{r e d}^{\text {field }}$, and an interaction part which vanishes if either the matter or the field variables vanish, $H_{r e d}^{i n t}$. Then we get

$$
H_{\text {red }}=H_{\text {red }}^{\text {mat }}\left[\varrho^{*}, \pi\right]+H_{\text {red }}^{i n t}\left[\varrho^{*}, \pi, h^{T T}, p^{T T}\right]+H_{\text {red }}^{\text {field }}\left[h^{T T}, p^{T T}\right] .
$$

By construction, $H_{\text {red }}$ is conserved in time.

The power supplied by the matter system into the field, or vice versa, is given by (the Hamiltonian which evolves the matter system is $\left.H_{r e d}^{\text {mat }}+H_{r e d}^{i n t}\right)$,

$$
\frac{d\left(H_{r e d}^{\text {mat }}(t)+H_{r e d}^{i n t}(t)\right)}{d t}=-\frac{d H_{r e d}^{f i e l d}(t)}{d t} .
$$

(Notice, $d_{t} H_{\text {red }}^{\text {field }}=-\partial_{t} H_{\text {red }}^{i n t}\left[\varrho^{*}, \pi, h^{T T}(t), p^{T T}(t)\right]$, which shows the importance of $H_{r e d}^{i n t}$ for the exchange of energy.)

The approximation of the balance equation (25) to first post-Newtonian order is given by, considering dissipative terms only,

$$
\frac{d H_{r e d}^{1 p N}(t)}{d t}=-\frac{d H_{r e d}^{r a d}(t)}{d t}
$$

where the post ${ }^{1}-N e w t o n i a n$ Hamiltonian $H_{\text {red }}^{1 p N}$ is identical with $H_{\text {red }}^{\operatorname{mat}(1 p N)}$ and where $H_{r e d}^{r a d}$ is given by equation (23). In some sense, $H_{\text {red }}^{\text {rad }}$ could be denoted by $H_{\text {red }}^{\text {field }(1 p N)}$. 
The dropped $H_{r e d}^{i n t}$ is of the order $(v / c)^{4}$; its leading order term reads,

$$
H_{r e d}^{i n t[4]}=-8 \pi G \int d^{3} x h_{i j}^{T T}\left[\frac{\pi_{i} \pi_{j}}{\varrho^{*}}+\frac{1}{4 \pi G} \partial_{i} U^{*} \partial_{j} U^{*}\right],
$$

where $U^{*}=\int d^{3} y \varrho^{*} /|x-y|$.

If we average the equation (26) over several periods of the emitted wave it turns out that only the wave zone contributes to the right side of the equation (26) if we postulate a quasi stationary process which lasts longer than it takes time for the wave to reach the wave zone. In the wave zone we may then use the homogeneous field equations which result from $H_{r e d}^{r a d}$, i.e.

$$
\frac{\partial h^{T T}}{\partial t}=2 p^{T T}, \frac{\partial p^{T T}}{\partial t}=\frac{c^{2}}{2} \nabla^{2} h^{T T}
$$

Assuming no-incoming radiation conditions, it is easily shown that the following relation holds,

$$
<\frac{d H_{r e d}^{r a d}(t)}{d t}>_{t}=\frac{8 \pi G}{c^{5}} \oint_{w . z .}<\left(\partial_{t} h^{T T}(t)\right)^{2}>_{t} r^{2} d \Omega,
$$

where $\langle\ldots\rangle_{t}$ denotes the time average over several periods of the wave. As a result we find that the enery loss in the matter system appears as radiation power in the wave zone (w.z.). Notice that through our assumption of quasi-stationarity we were able to treat the whole radiation emission problem in a small sandwich (with a width of a few periods of the wave) of space-like hypersurfaces. As in the wave zone the Hamiltonian of the gravitational field is always given by $H_{r e d}^{r a d}$ the generalization of our energy balance treatment to the full dynamics is straightforward.

For the losses of linear momentum and angular momentum similar considerations hold. Here, corresponding to equation (25), the time derivative of the momentum constraint (8) comes in. The explicit wave zone expressions can be found, e.g. in [31].

5. Post ${ }^{2.5}$-Newtonian two-body point-mass Hamiltonian. The simplest model for an astrophysical two-body system are two non-spinning point-like masses interacting gravitationally. Although point-like objects do not exist in general relativity - black holes are the most compact objects, and they are extended - the model can be used in approximate calculations as long as no inconsistencies occur. Calculations have shown that the property of being "point-like" might already be invalidaded at the post ${ }^{3}$-Newtonian order, [21], latest, however, at post ${ }^{5}-$ Newtonian order, [8]. It is a remarkable property of the Einstein theory that up to the post ${ }^{2.5}$-Newtonian approximation, as well as at the post $^{3.5}$-Newtonian order, well-separated compact objects can be treated as "point-like" with only mass parameters entering the equations of their external motion. This property is sometimes called the "effacement" of the internal structure in the external problem, [8], [9].

The point-mass model results from the fluid-matter model in putting $\Pi=p=0$ and setting $\varrho^{*}(t, x)=\Sigma_{a} m_{a} \delta^{3}\left(x-x_{a}(t)\right)$ and $\pi_{i}(t, x)=\Sigma_{a} p_{a i} \delta^{3}\left(x-x_{a}(t)\right)$, where $m_{a}, x_{a}^{i}$, and $p_{a i}$ are mass parameter, position vector, and linear momentum of body $a$, respectively. After regularization of the gravitational potentials and of the Hamiltonian, respectively the equations of motion, it turns out that $m_{a}$ are the rest masses of the bodies. Powerful 
methods to achieve the regularization are Hadamard's "partie finie" and Riesz's analytic continuation procedure; for applications, e.g. see [25] and [8], respectively; for details, see the contribution by P. Jaranowski, in these proceedings.

For point masses the equation of motion (5) and (6) translate, respectively, into the equations

$$
\begin{aligned}
\frac{d x_{a}^{i}}{d t} & =\frac{\partial H}{\partial p_{a i}}, \\
\frac{d p_{a i}}{d t} & =-\frac{\partial H}{\partial x_{a}^{i}} .
\end{aligned}
$$

In the coordinate system introduced above, the Hamiltonian $H_{r e d}^{\text {mat }}+H_{r e d}^{\text {int }}$, up to the post $^{2}$-Newtonian approximation, appears as follows, the additive constant $\left(m_{1}+m_{2}\right) c^{2}$ being dropped, [12],

$$
\begin{aligned}
& H_{r e d}^{2 p N}\left(x, p_{1}, p_{2}\right)=\frac{1}{2}\left(\frac{p_{1}^{2}}{m_{1}}+\frac{p_{2}^{2}}{m_{2}}\right)-\frac{G m_{1} m_{2}}{r}-\frac{1}{8 c^{2}}\left(\frac{p_{1}^{4}}{m_{1}^{3}}+\frac{p_{2}^{4}}{m_{2}^{3}}\right) \\
& +\frac{G^{2}\left(m_{1}+m_{2}\right) m_{1} m_{2}}{2 c^{2} r^{2}}+\frac{G m_{1} m_{2}}{8 c^{2} r}\left[-12\left(\frac{p_{1}^{2}}{m_{1}^{2}}+\frac{p_{2}^{2}}{m_{2}^{2}}\right)+28 \frac{\left(p_{1} \cdot p_{2}\right)}{m_{1} m_{2}}\right. \\
& \left.+4 \frac{\left(n \cdot p_{1}\right)\left(n \cdot p_{2}\right)}{m_{1} m_{2}}\right]+\frac{1}{16 c^{4}}\left(\frac{p_{1}^{6}}{m_{1}^{5}}+\frac{p_{2}^{6}}{m_{2}^{5}}\right)-\frac{G^{3} m_{1} m_{2}\left(m_{1}^{2}+m_{2}^{2}+5 m_{1} m_{2}\right)}{4 c^{4} r^{3}} \\
& +\frac{G m_{1} m_{2}}{8 c^{4} r}\left[5\left(\frac{p_{1}^{4}}{m_{1}^{4}}+\frac{p_{2}^{4}}{m_{2}^{4}}\right)-11 \frac{p_{1}^{2} p_{2}^{2}}{m_{1}^{2} m_{2}^{2}}-2 \frac{\left(p_{1} \cdot p_{2}\right)^{2}}{m_{1}^{2} m_{2}^{2}}\right. \\
& +5 \frac{p_{1}^{2}\left(n \cdot p_{2}\right)^{2}+p_{2}^{2}\left(n \cdot p_{1}\right)^{2}}{m_{1}^{2} m_{2}^{2}}-12 \frac{\left(p_{1} \cdot p_{2}\right)\left(n \cdot p_{1}\right)\left(n \cdot p_{2}\right)}{m_{1}^{2} m_{2}^{2}} \\
& \left.-3 \frac{\left(n \cdot p_{1}\right)^{2}\left(n \cdot p_{2}\right)^{2}}{m_{1}^{2} m_{2}^{2}}\right]+\frac{G^{2} m_{1} m_{2}}{4 c^{4} r^{2}}\left[m_{2}\left(10 \frac{p_{1}^{2}}{m_{1}^{2}}+19 \frac{p_{2}^{2}}{m_{2}^{2}}\right)\right. \\
& \left.+m_{1}\left(10 \frac{p_{2}^{2}}{m_{2}^{2}}+19 \frac{p_{1}^{2}}{m_{1}^{2}}\right)-\left(m_{1}+m_{2}\right) \frac{27\left(p_{1} \cdot p_{2}\right)+6\left(n \cdot p_{1}\right)\left(n \cdot p_{2}\right)}{m_{1} m_{2}}\right]
\end{aligned}
$$

where $x=x_{1}-x_{2}, r=|x|$, and $n=x / r$ hold. For the obtention of the Hamiltonian (32) the solution of the equation for the field variable $h^{T T}$ was needed in leading order, [22].

We remark that in harmonic coordinates, at the post ${ }^{2}$-Newtonian approximation, an ordinary Hamiltonian is not achieveable. In harmonic coordinates the two-body dynamics is most easily formulated on the equations of motion level ([8], [21]). At the post $^{2}$-Newtonian level of approximation the corresponding Lagrangian contains higher time derivatives of the position variables [8], [11], [16], [10]. This leads to a constraint Hamiltonian system, e.g. see [13].

The near-zone expansion of $h^{T T}$ to order $1 / c$ yields the part of the Hamiltonian which, to leading order, is responsible for the back-reaction of the emitted gravitational waves. It takes the form, [28],

$$
H_{\text {red }}^{\text {reac }}\left(x, p_{a}, t\right)=-\frac{16 \pi G}{c^{4}} h_{i j}^{T T(r e a c)}(t)\left(\frac{p_{1 i} p_{1 j}}{2 m_{1}}+\frac{p_{2 i} p_{2 j}}{2 m_{2}}-G m_{1} m_{2} \frac{x^{i} x^{j}}{2 r^{3}}\right),
$$


where $a=1,2$ and where $h^{T T(\text { reac })}$ reads

$$
h_{i j}^{T T(r e a c)}(t)=-\frac{1}{20 \pi c} \frac{d^{3}}{d t^{3}} Q_{i j}(t) .
$$

$Q_{i j}$ is the usual Newtonian mass-quadrupole tensor for the matter system, $Q_{i j}=m_{1}\left(x_{1}^{i} x_{1}^{j}\right.$ $\left.-(1 / 3) \delta_{i j}\left|x_{1}\right|^{2}\right)+m_{2}\left(x_{2}^{i} x_{2}^{j}-(1 / 3) \delta_{i j}\left|x_{2}\right|^{2}\right)$. The dissipation property of the reaction Hamiltonian, equation (33), has its origin in the time-asymmetry of $h^{T T \text { (reac) }}$.

The reaction Hamiltonian adds up with the post ${ }^{2}$-Newtonian Hamiltonian, equation (32), to give the post ${ }^{2.5}$-Newtonian two-body Hamiltonian,

$$
H_{\text {red }}^{2.5 p N}\left(x, p_{a}, t\right)=H_{r e d}^{2 p N}\left(x, p_{a}\right)+H_{r e d}^{r e a c}\left(x, p_{a}, t\right),
$$

where $a=1,2$. The Hamiltonian $H^{2.5 p N}$ describes the motion of a two-body point-mass system in general relativity up to the first radiation reaction level.

Explicit solutions for the dynamics defined by the conservative Hamiltonian (32) are given in [12], [29], and [32]. The energy and angular momentum losses related with the dissipative Hamiltonian (33) have been treated in [23] already many years ago. Energy and angular momentum losses in general binary systems are known up to the order $1 / c^{7}$, [6], [20]. Recently, for circular orbits, energy losses at the orders $1 / c^{9},[5]$, and $1 / c^{10}$, [4], have been obtained. The two-body equations of motion are known in the dissipative part up to the post ${ }^{3.5}$-Newtonian level, [18]; the dissipative n-body Hamiltonian at the post $^{3.5}$-Newtonian level has been derived only quite recently, [19].

\section{References}

[1] V. I. Arnold, Sur la géometrie differentielle des groupes de Lie de dimension infinie et ses applications a l'hydrodynamique des fluids parfaits, Ann. Inst. Fourier (Grenoble) 16 (1966), 319-361.

[2] R. Arnowitt, S. Deser, and C. M. Misner, Gravitational-electromagnetic coupling and the classical self-energy problem, Phys. Rev. 120 (1960), 313-320.

[3] R. Arnowitt, S. Deser, and C. M. Misner, The dynamics of general relativity, in: Gravitation: An Introduction to Current Research, L. Witten (ed.), Wiley, New York 1962, 227-265.

[4] L. Blanchet, Energy losses by gravitational radiation in inspiralling compact binaries to five halves post-Newtonian order, Phys. Rev. D 54 (1996), 1417-1438.

[5] L. Blanchet, T. Damour, B. R. Iyer, C. M. Will, and A. G. Wiseman, Gravitationalradiation damping of compact systems to second post-Newtonian order, Phys. Rev. Lett. 74 (1995), 3515-3518.

[6] L. Blanchet and G. Schäfer, Higher-order gravitational radiation losses in binary systems, Mon. Not. R. astr. Soc. 239 (1989), 845-867; and Erratum: Mon. Not. R. astr. Soc. 242 (1990), 704.

[7] L. Blanchet and G. Schäfer, Gravitational wave tails and binary star systems, Class. Quantum Grav. 10 (1993), 2699-2721.

[8] T. Damour, Gravitational radiation and the motion of compact objects, in: Gravitational Radiation, N. Deruelle and T. Piran (eds.), North-Holland Publishing, Amsterdam 1983, 59-144. 
[9] T. Damour, The problem of motion in Newtonian and Einsteinian gravity, in: 300 Years of Gravitation, S. W. Hawking and W. Israel (eds.), Cambridge University Press, Cambridge 1987, 128-198.

[10] T. Damour, L. P. Grishchuk, S. M. Kopejkin, and G. Schäfer, Higher-order relativistic dynamics of binary systems, in: Proc. 5th Marcel Grossmann Meeting on General Relativity, The University of Western Australia 1988, D. G. Blair and M. J. Buckingham (eds.), World Scientific, Singapore 1989, 451-459.

[11] T. Damour and G. Schäfer, Lagrangians for $n$ point masses at the second postNewtonian approximation to general relativity, General Relativity and Gravitation 17 (1985), 879-905.

[12] T. Damour and G. Schäfer, Higher-order relativistic periastron advances and binary pulsars, Nuovo Cimento B 101 (1988), 127-176.

[13] T. Damour and G. Schäfer, Redefinition of position variables and the reduction of higher order lagrangians, Journ. Math. Phys. 32 (1991), 127-134.

[14] B. S. DeWitt, Quantum theory of gravity. I. The canonical theory, Phys. Rev. 160 (1967), 1113-1148.

[15] J. Ehlers, Über den Newtonschen Grenzwert der Einsteinschen Gravitationstheorie, in: Grundlagenprobleme der modernen Physik, J. Nitsch, J. Pfarr, and E.-W. Stachow (eds.), Bibliographisches Institut, Mannheim 1981, 65-84.

[16] L. P. Grishchuk and S. M. Kopejkin, Equations of motion for isolated bodies with relativistic corrections including the radiation reaction force, in: Relativity in Celestial Mechanics and Astrometry, J. Kovalevsky and V. A. Brumberg (eds.), Reidel, Dordrecht 1986, 19-34.

[17] D. D. Holm, Hamiltonian formalism for general-relativistic adiabatic fluids, Physica 17D (1985), 1-36.

[18] B. R. Iyer and C. M. Will, Post-Newtonian gravitational radiation reaction for two-body systems: Nonspinning bodies, Phys. Rev. D 52 (1995), 6882-6893.

[19] P. Jaranowski and G. Schäfer, Radiative 3.5 post-Newtonian ADM Hamiltonian for many-body point-mass systems, Phys. Rev. D (1996), submitted.

[20] W. Junker, G. Schäfer, Binary systems: higher order gravitational radiation damping and wave emission, Mon. Not. R. astr. Soc. 254 (1992), 146-164.

[21] S. M. Kopejkin, General-relativistic equations of binary motion for extended bodies, with conservative corrections and radiation damping, Sov. Astron. 29 (1985), 516-524.

[22] T. Ohta, H. Okamura, T. Kimura, and K. Hiida, Coordinate condition and higher order gravitational potential in canonical formalism, Progress of Theoretical Physics 51 (1974), 1598-1612.

[23] P. C. Peters, Gravitational radiation and the motion of two point masses, Phys. Rev. 136 (1964), B1224-B1232.

[24] T. Regge and T. Teitelboim, Role of surface integrals in the Hamiltonian formulation of general relativity, Annals of Physics 88 (1974), 286-318.

[25] G. Schäfer, The gravitational quadrupole radiation-reaction force and the canonical formalism of ADM, Ann. Phys. (N.Y.) 161 (1985), 81-100.

[26] G. Schäfer, Higher-order post-Newtonian hydrodynamics, in: Proc. 5th Marcel Grossmann Meeting on General Relativity, The University of Western Australia 1988, D. G. Blair and M. J. Buckingham (eds.) World Scientific, Singapore, 1986, 467-470.

[27] G. Schäfer, Reduced Hamiltonian formalism for general-relativistic adiabatic fluids and applications, Astron. Nachrichten 311 (1990), 213-217. 
[28] G. Schäfer, The general relativistic two-body problem. Theory and experiment, in: Symposia Gaussiana, Proc. 2nd Gauss Symposium, Conf. A: Mathematical and Theoretical Physics, Munich 1993, M. Behara, R. Fritsch, and R. G. Lintz (eds.), Walter de Gruyter, Berlin 1995, 667-679.

[29] G. Schäfer and N. Wex, Second post-Newtonian motion of compact binaries, Phys. Lett. A 174 (1993), 196-205; and Erratum: Phys. Lett. A 177 (1993), 461.

[30] K. Sundermeyer, Constraint Dynamics, Lecture Notes in Physics 169, Springer-Verlag, Berlin 1982.

[31] K. S. Thorne, Multipole expansion of gravitational radiation, Rev. Mod. Phys. 52 (1980), 299-339.

[32] N. Wex and R. Rieth, The solution of the second post-Newtonian two-body problem, in: Symposia Gaussiana, Proc. 2nd Gauss Symposium, Conf. A: Mathematical and Theoretical Physics, Munich 1993, M. Behara, R. Fritsch, and R. G. Lintz (eds.), Walter de Gruyter, Berlin 1995, 681-693. 